\title{
Visualization of Secondary Flow Development in High Aspect Ratio Channels with Curvature
}

Michael L. Meyer

Lewis Research Center

Cleveland, Ohio

and

James E. Giuliani

Ohio Aerospace Institute

Brook Park, Ohio

Prepared for the

30th Joint Propulsion Conference

cosponsored by the AIAA, ASME, SAE, and ASEE

Indianapolis, Indiana, June 27-29, 1994

$$
\overbrace{0}^{m}
$$
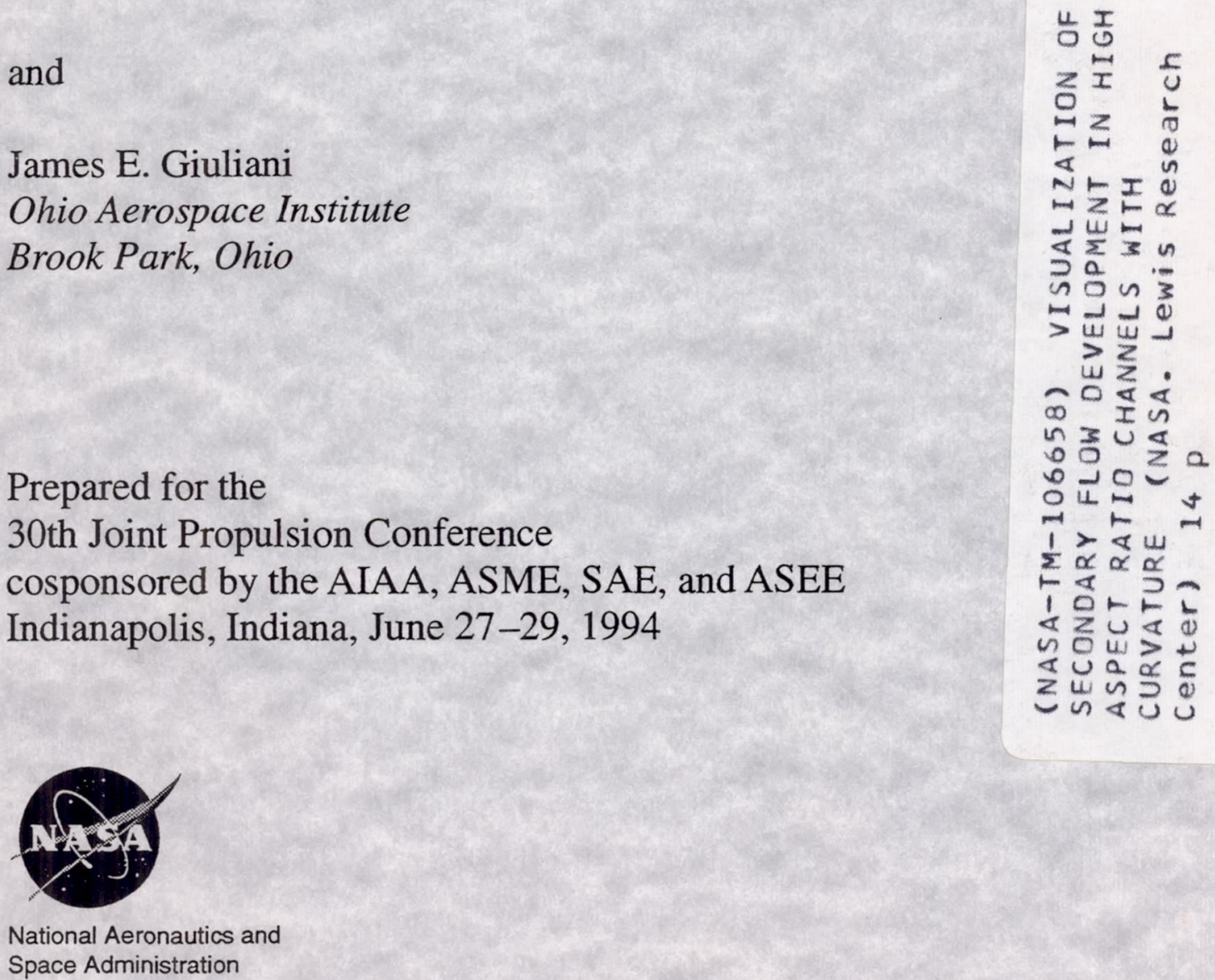

National Aeronautics a 


\title{
VISUALIZATION OF SECONDARY FLOW DEVELOPMENT IN HIGH ASPECT RATIO CHANNELS WITH CURVATURE
}

\author{
Michael L. Meyer \\ NASA Lewis Research Center \\ Cleveland, Ohio 44135 \\ James E. Giuliani* \\ Ohio Aerospace Institute \\ Brook Park, Ohio 44142
}

\begin{abstract}
$\underline{\text { Abstract }}$
The results of an experimental project to visually examine the secondary flow structure that develops in curved, high aspect-ratio rectangular channels are presented. The results provide insight into the fluid dynamics within high aspect ratio channels. A water flow test rig constructed out of plexiglass, with an adjustable aspect ratio, was used for these experiments. Results were obtained for a channel geometry with a hydraulic diameter of 10.6 $\mathrm{mm}(0.417 \mathrm{in}$.), an aspect ratio of 5.0, and a hydraulic radius to curvature radius ratio of 0.0417 . Flow conditions were varied to achieve Reynolds numbers up to 5,100. A new particle imaging velocimetry technique was developed which could resolve velocity information from particles entering and leaving the field of view. Time averaged secondary flow velocity vectors, obtained using this velocimetry technique, are presented for $30^{\circ}, 60^{\circ}$, and $90^{\circ}$ into a $180^{\circ}$ bend and at a Reynolds number of 5,100. The secondary flow results suggest the coexistence of both the classical curvature induced vortex pair flow structure and the eddies seen in straight turbulent channel flow.

\section{Introduction}

Interest in High Aspect Ratio Cooling Channels (HARCC) for rocket engine thrust chambers has created a need to better understand the impact of channel aspect ratio on coolant fluid dynamics. Tests by Carlile and Quentmeyer [1] have demonstrated the benefits of HARCC for straight cooling passages. In an actual contoured engine, however, the effect of aspect ratio on the "curvature effect" has not been established. For tubes of circular and square cross-section, the development of strong secondary flows in curves,
\end{abstract}

the "curvature effect", has been well documented (see for example Schlichting [2] and Humphrey and Launder [3]). The higher momentum fluid in the center of the tube experiences greater centrifugal acceleration than the fluid near the walls. Thus, a secondary flow develops with the centerline fluid moving toward the outer wall of the bend, and the outer fluid moving toward the inner wall. A pair of vortices, symmetric about the plane of curvature, is formed.

In a rocket engine cooling passage, the heat transfer to the coolant is significantly enhanced in the throat section of the chamber, where the curvature induced secondary flow causes the cooler centerline fluid to flow to the hot wall. Hendricks and Simon [4] showed that the heat transfer enhancement may be as much as $40 \%$. Since the throat is the region of highest heat flux from the chamber, this effect is critical for maintaining a safe throat wall temperature. As channel aspect ratio is increased, the secondary flow vortices are stretched and the vortex core begins to form a shear layer. This stretching of the vortex flow structure may weaken it or even cause it to break down. A numerical study by Kacynski [5] postulated that reduced mixing in a coolant channel with an aspect ratio of eight will result in significantly higher wall temperatures than an identical channel with well mixed coolant, due to thermal stratification in the coolant. In such a case, the benefits of the high aspect ratio geometry would be reduced. Other numerical studies, such as Yagley et al.[6] and Lebail and Popp [7], have investigated high aspect ratio rocket engine coolant channels, but these efforts have been hampered by a lack of sufficient validating data.

A limited amount of relevant experimental

\footnotetext{
* Member AIAA

"Copyright @ 1994 by the American Institute of Aeronautics and Astronautics, Inc. No copyright is asserted in the United States under Title 17, U.S. Code. The U.S. Government has a royalty-free license to exercise all rights under the copyright claimed herein for Governmental purposes. All other rights are reserved by the copyright owner."
} 
data for validating the numerical methods is available. Wall temperature data is available from hot-fire engine tests for channels without curvature and aspect ratio $=5.0$ in the report by Carlile and Quentmeyer [1]. More detailed fluid dynamic data are available for unheated square and circular cross section channels with curvature at Reynolds numbers up to 40,000 from Taylor et al. [8], but a better understanding of the fundamental flow phenomena in high aspect ratio channels is needed to fully evaluate the benefits of this geometry.

With the advancement of electronic image processing methods and recording techniques, flow visualization has become a powerful tool for velocity measurements that can be applied to a curving high aspect ratio channel. Imaging the trajectories of illuminated particles in a seeded flow can provide both a qualitative description of the velocity flow field and quantitative measurements of the flow velocity. Many different methods have evolved in the attempt to obtain flow field velocity measurements from flow field images. In some previous velocimetry imaging work by Marko and Rimai [9], video data containing particle streaks were digitally recorded and then processed manually at a later time. To determine the particle streak endpoints and flow velocity direction, single frames of the recorded data were displayed on a computer screen and an operator would manually mark the starting and ending points of the streaks with a "mouse" or similar pointing device. Khalighi and Lee [10] automated this process using computer programs to process particle images and extract velocity information. Many of the systems reviewed, however, require that the visualized area be a closed system and that no particles leave or enter the field under study (see Wernet [11] for example).

To investigate the effects of channel aspect ratio on secondary flow and to qualitatively and quantitatively provide secondary velocity fields for anchoring numerical codes, a flow visualization/particle imaging velocimetry experiment was initiated at the NASA Lewis Research Center. A new technique was developed, which uses computer algorithms to resolve qualitative and quantitative flow field velocity information from particle streaks recorded in a time sequence of digitized, single exposure video images. The computer algorithms developed for this project automatically resolve the cross-plane velocity information perpendicular to the bulk flow velocity direction from particle streaks entering and leaving the field of view. The results of applying this technique to secondary flow field video data, obtained from an aspect ratio 5.0 curved channel with turbulent water flow, are presented in this report.

\section{Experimental Apparatus}

The experimental test rig, shown in figure 1, was designed to permit visualization of the secondary flow structure that develops in a curved rectangular channel. The test rig consisted of a curved plexiglass duct, a bubble generator, a copper vapor laser system, a video camera and recorders, and a refraction correcting window chamber.

The test channel was constructed from upper and lower plexiglass plates, which form the channel top and bottom, and two strips of plexiglass which make up the side walls. The plexiglass strips fit into grooves cut into the upper and lower plates. The grooves are spaced $31.75 \mathrm{~mm}$ (1.25 in.) apart and provide a constant channel width. Wall strips of different heights may be placed into the grooves to change the height of the channel. Aspect ratios from 1.0 to 5.0 are possible and a wall height was selected for this experiment that gave the channel an aspect ratio of 5.0. The total length of the channel is $3.44 \mathrm{~m}(11.3 \mathrm{ft}$.) and consists of two 1.52 $\mathrm{m}\left(5.0 \mathrm{ft}\right.$.) straight sections and a $180^{\circ}$ bend in the center. Upon entering the channel, the flow first passes through a $1.52 \mathrm{~m}$ (5.0 ft.) straight section where disturbances dissipate and the flow is allowed to develop. The channel then turns $180^{\circ}$ with a 127 $\mathrm{mm}$ (5.0 in.) centerline radius of curvature. The channel continues straight for an additional $1.52 \mathrm{~m}$ $(5.0 \mathrm{ft}$.) to the flow outlet. Water is forced through the system in an open loop configuration by the domestic water supply pressure. Reynolds numbers based on hydraulic diameter $\left(\mathrm{Re}_{\mathrm{dh}}\right)$ up to 40,000 can be obtained.

To visualize the flow structure in the curved section, the hydrogen bubble technique, as described by Davis and Fox [12] and Tory and Haywood [13], is used to seed the flow. With this technique, hydrogen bubbles are formed on thin tungsten wires placed within the flow field. The wires form the cathode (negative) pole of a direct current (DC) circuit, and an additional conductor forms the anode (positive) pole. When a current is applied to the circuit, electrolysis takes place forming oxygen on the anode and hydrogen on the cathode. As hydrogen bubbles form on the cathode wires, the 
flow strips them away.

In the tests reported here, five wires spanned the channel vertically, spaced evenly across the width of the channel and $25.4 \mathrm{~mm}$ (1.0 in.) upstream of the turning section. Three alternating wires were used as cathodes, and the remaining two wires were used as the anodes. The power supply used to generate the hydrogen bubbles provided 160 volts DC and was limited to $100 \mathrm{~mA}$ of current. Wire diameters of $0.127 \mathrm{~mm}$ (0.005 in.) and 0.254 $\mathrm{mm}(0.010 \mathrm{in}$.) were used for the cathodes and anodes respectively.

A light sheet was used to illuminate the hydrogen bubbles from above the rig as shown in figure 1. To generate the light sheet, the output beam from a copper vapor laser was directed through an optical train similar to that described by Diemunsch and Prenel [14]. The resulting light sheet (or column) had a cross-section of $25.4 \mathrm{~mm}$ by $12.7 \mathrm{~mm}$ (1.0 in. by $0.5 \mathrm{in}$.) and passed completely through the test rig in the vertical direction. The width of the column could not be adjusted wider to illuminate the entire $31.75 \mathrm{~mm}$ (1.25 in.) width of the channel due to the diameters of the optical components. Therefore, the $6.35 \mathrm{~mm}$ ( $0.25 \mathrm{in}$.) nearest the inner wall of the channel was not visualized. This did not compromise the investigation since preliminary tests indicated that the most significant secondary flows were near the opposite (outer) wall. The light sheet thickness was selected such that the seedant bubble residence time would be sufficient to identify and measure small secondary velocities. With a sheet thickness of 12.7 $\mathrm{mm}(0.5 \mathrm{in}$.), secondary velocities as low as $1 \%$ of the bulk streamwise velocity could be ascertained, although a limit of $3 \%$ was set during the data analysis.

A video camera was used to capture the illuminated particles streaks. The camera signal was sent to both a standard VHS video recorder and a $19 \mathrm{~mm}$ (3/4 in.) tape video recorder. By positioning the camera perpendicular to the light sheet as shown in figure 1 , only motion in the cross plane was observed.

A triangular optical compensating window chamber, seated against the outer wall and filled with water, minimized the image distortion caused by focussing the camera through a curved plexiglass side wall. The side of the window chamber provided a flat surface parallel to the focus plane, and the water filled cavity provided a more closely matched index of refraction at the curved sidewall. This optical compensating window was sealed to the test rig with a silicone adhesive and could be removed, cleaned and re-attached at a different location. For the tests reported here, the optical compensating window was repositioned such that video image data were recorded at $30^{\circ}, 60^{\circ}$, and $90^{\circ}$ into the bend.

\section{Particle Imaging Velocimetry}

To resolve flow patterns and velocity information, a computer program was developed that analyzes hydrogen bubble streaks recorded in sequential images of digitized video data. The analysis of flow visualization images consists of five steps which include acquisition and preprocessing, calculation of bubble streak centroids, extraction of streak endpoints, multiple frame search to find vectors and computation of vector magnitude and direction.

For the present system configuration, a copper vapor laser was used to generate a light sheet and a video camera recorded the illuminated particle streaks. To prepare the data for analysis, the video tape was played back into a digitizer frame buffer system which extracted sequential video fields at a framing rate of 60 fields per second. Each digitized image, or field, was 720 pixels wide and 240 pixels high with each pixel having a luminance value from 0 to 255 . The digital images were then stored on a computer hard disk for later processing. A detailed description of the digitizing process is given by Marko and Rimai [9].

To begin the analysis, the program reads in sequential images in time and preprocesses the data. The first step of preprocessing is to improve the effectiveness of the particle streak recognition routines by passing a median histogram over the image, to smooth the gradients between dark and light areas of the image. Then an image, which was recorded before the hydrogen bubbles were introduced into the flow and hence contains no streaks, is subtracted from the data image to mask out glare and other visual obstructions. Figure 2 shows a typical image of digitized video data. Figure 3 shows the same image after preprocessing to improve image quality. In the resulting image, the brightness of each pixel can be thought of as an elevation corresponding to an $\mathrm{X}$ and $\mathrm{Y}$ coordinate position. The centerline of the streak can then be 
thought of as the 'peaks' or 'ridges' in this 3-D space.

Particle streaks are resolved from individual images by first calculating the centroids of all streaks. Particle streak centroids are calculated by integrating the luminance gradient over a specific range of image pixels. The luminance gradient is formed by two neighboring pixels with different levels of brightness. First the program scans down vertical lines and then across horizontal lines. Integration starts when the gradient switches from negative to positive, and stops at the next occurrence of a negative to positive switch. If the integral was above a selected threshold, indicating that the integral has probably passed over a streak, and a centroid is marked at the point of maximum brightness over the range of integration.

Once all possible centroids in the image have been identified, a search algorithm is employed to resolve the endpoints from the calculated centroids. First the program scans down vertical lines and then across horizontal lines. When a centroid and hence a possible streak is encountered, the search algorithm is called. This routine uses a regressive backtracking technique to search for neighboring centroids. When all centroids have been found, the distribution of centroids is examined, endpoints are resolved and a magnitude of the possible streak is calculated. To reduce the probability of detecting a false streak, endpoints are not recorded if the magnitude is smaller than 8 pixels, or about $3 \%$ of the bulk streamwise velocity. If the magnitude is greater than $3 \%$ of the bulk streamwise velocity, the endpoints are recorded using a data encoding technique which allows one endpoint of a streak to be calculated given the $\mathrm{X}, \mathrm{Y}$ coordinates of the other endpoint. Figure 4 shows the bubble streaks calculated from the image in figure 2 .

To resolve velocity vectors, the vector resolution routine searches for particle streaks that exist in the light sheet for two or three successive frames. The routine stores three images in memory; Image \#1 at time t, Image \#2 at time $t+1 / 60$, and Image \#3 at time $t+1 / 30$. Image \#1 is searched until a particle streak endpoint is found. This point is flagged as the tail of a vector. The $\mathrm{X}, \mathrm{Y}$ coordinates of the corresponding streak endpoint are obtained from the data encoding technique and a circular search region with a radius of 3 pixels is defined around this point. This region is then searched in Image \#2, which is the next image in time at $t+1 / 60$ seconds. If an endpoint is found within this search region, then the coordinates of its corresponding endpoint are calculated. This point is flagged as the head of a vector. It is then inferred that the particle entered the light sheet at the position marked as the tail of the vector at time $t$, traveled in a direction taking it through the two endpoints that were matched up, and then left the light sheet at the position marked as the head of the vector at time $t+1 / 30$. This search is carried out for a third field in the event a particle's residence time in the light sheet is greater than the 1/30th of a second represented by two images.

The velocity scale for measurement of the cross-plane velocity is calculated from the channel geometry and the bulk velocity of the flow. The vertical dimension of the rectangular channel was $6.4 \mathrm{~mm}$ ( $0.25 \mathrm{in}$.) high. This corresponded to approximately 102 pixels in the digitized video image. The light sheet created by the laser is 12.7 $\mathrm{mm}(0.5 \mathrm{in}$.) thick in the direction of the bulk flow velocity. The time a particle is in the light sheet is given by:

$$
t_{\text {illuminated }}=\frac{\text { light sheet thickness }}{\text { bulk flow velocity }}=\frac{0.0127(\mathrm{~m})}{U(\mathrm{~m} / \mathrm{s})}
$$

Once the illuminated time is known and the particle cross-plane displacement is known, calculated from the streak endpoints, the cross-plane velocity can be calculated.

$$
V_{\text {crass plane }}=\frac{\text { particle displacement }}{t_{\text {illuminated }}} * \frac{.0064(m)}{102 \text { pixels }}
$$

To enhance the information conveyed by the computed velocity vectors, an interpolation algorithm is used to transform the random distribution of velocity vectors to a uniform grid of the flow field as was done by Wernet [11] and Foley [15]. The interpolated flow field represents the time averaged secondary velocity. The routine starts by generating a uniform grid from a specified number of divisions. Looping through each grid point, the algorithm sorts the raw velocity vector data according to the distance away from the current grid point. The 15 nearest raw vectors are used in a weighted least squares analysis. A planar surface is fit to the velocity vector data, and used to interpolate the velocity vector magnitude and 
direction at the specified grid point. The weighting factor used in the analysis is the inverse distance from the grid point. The distribution of the raw velocity vectors strongly affects the quality of the interpolation. When the data density in the region of the interpolated grid point is high, a good estimate of the local velocity vector is obtained.

\section{Sources of Error}

Several potential sources for error were present within the methodology employed for obtaining the velocity fields. Two potential errors arose because of the finite thickness of the light sheet in the direction of the bulk flow velocity. One problem was that even if there were no secondary flows (i.e. if the flow followed paths of solid body rotation), the bubbles would leave small radial velocity streaks due to the channel curvature within the $12.7 \mathrm{~mm}$ ( $0.5 \mathrm{in}$.) light sheet. This false velocity was $1 \%$ of the bulk streamwise velocity, but because the streak selection criteria did not accept streaks less than $3 \%$ of the bulk streamwise velocity, the effect was reduced. The false velocity could still be superimposed on the actual velocities, but false streaks due only to curvature would not be picked up. The second problem with using a finite thickness light sheet was that the results, which are presented for a single point in the bend, actually represent a six degree segment, and the flow could continue to develop through the light sheet.

Another type of error arose when applying the PIV technique. Determining flow direction relied on matching streak endpoints in successive frames and assumed that the second streak was a continuation of the first one. It was possible that two independent streaks could occur in such a manner that their endpoints matched in successive frames by coincidence. To minimize this type of error, the seedant density was kept low for the imaging analysis runs, which reduced the possibility of having streaks overlap. Also, since the boundary layer inside the channel will decrease the local flow velocity near the wall relative to the bulk flow velocity, the calculation of $t_{\text {illuminated }}$ from equation (1) for the cross-plane velocity could be under-estimated for streaks resolved near the walls.

An additional source of error was present when applying the interpolation routine for calculating a time averaged velocity field. The interpolation algorithm interpolates a vector at a given position based on the 15 closest vectors. If the density of vectors is low in a particular region, the interpolation routine may not accurately predict the actual flow velocity at that point because the vectors used in the calculation are not close to the region of interest.

\section{Discussion of Results}

Flows were observed and recorded for $\mathrm{Re}_{\mathrm{dh}}$ ranging from 350 to 37,500 . Quantitative results, however, are presented only at $\mathrm{Re}_{\mathrm{dh}}=5,100$ because the framing rate of the video system was not fast enough to apply the vector resolution technique at higher flowrates. Qualitative results are presented for lower Reynolds numbers.

Figures 5 to 7 present single frame images, taken from the video tape, of flow conditions ranging from laminar through turbulence transition. In the figures, the primary flow was out of the page and the inner channel wall (and center of curvature) are to the right. Note that the bubbles at the low flow rates leave only very small streaks, so it is more useful to look at the overall bubble patterns. All three images were taken at $90^{\circ}$ into the bend and a high bubble density.

Figure 5 shows the bubble pattern observed at $\operatorname{Re}_{\mathrm{dh}}=1,000$. Since this flow was laminar, the bubbles were not dispersed throughout the channel but remained in continuous sheets stripped from the generating wires. These sheets are bent by the secondary flows into the pattern in the figure. The direction of bubble motion was obtained by observing the video tape in slow motion. When animated, bubbles in the solid line across the center of the channel can be seen flowing towards the outer wall. The angle in the upper half of the channel has a symmetric counterpart in the lower half of the channel. The branches which extend from these angles toward the center of the channel are made up of bubbles moving toward the outer wall, and the branches near the upper and lower surfaces have bubbles moving toward the inner wall. The cusps of the angles represent the turn around point for the flow. Taken together, the flow exhibits the classical curvature effect vortex pair.

When the flow rate was increased to $\mathrm{Re}_{\mathrm{dh}}$ $=1,700$, figure 6 , the bubble pattern became more complicated. A smaller vortex began to develop within the larger upper vortex. There were not enough bubbles visible in the lower half of the channel to determine if a symmetric vortex forms. 
At $\mathrm{Re}_{\mathrm{dh}}=3,400$, figure 7, a strong, smaller scale, flow structure had developed. The structure appears similar to a vortex street, with offset pairs of smaller vortices, but this single frame image represents only one instant in time. Viewing the flow in real time, the vortex structure seen in the image was unstable and oscillated in a random fashion. However, even with this complicated flow pattern, the strong central current toward the outer wall and the upper and lower flows toward the inner wall remained.

Figures 8 to 10 contain raw velocity vectors calculated from sequential images of experimental video data at $\mathrm{Re}_{\mathrm{dh}}=5,100$ and $30^{\circ}, 60^{\circ}$, and $90^{\circ}$ into the bend respectively. The images and primary flow were oriented the same for these figures as for figures 5 to 7 . The video data for the $60^{\circ}$ case contained an unusual amount of glare around the outside edge of the channel which washed out particle streaks in this region. A vector representing $10 \%$ of the bulk flow velocity is placed at the top of each plot for scale, and lines are drawn outlining the walls of the channel.

These figures represent all of the vectors, individually calculated from 2 or 3 sequential frames, observed over a time span of several seconds. No averaging or smoothing is performed. At this $\mathrm{Re}_{\mathrm{dh}}$, the flow was turbulent and the bubbles have been distributed throughout the channel, and it became difficult to qualitatively evaluate the flow directly from observation. Some hydrogen bubble streaks represented momentary fluctuation of the flow and highlighted the flow's unsteady nature. Although the vector pattern appeared somewhat random, the flow was turbulent and so the random behavior was expected.

Figures 11 to 13 represent the results of interpolating the vectors in figures 8 to 10 to obtain time averaged velocity fields. Despite the apparent randomness of the vectors in the raw vector plots, the mean flow structure is clear in the interpolated plots, and the classical secondary flow can be seen in each figure, even at just 30 degrees into the bend. It should be re-emphasized that interpolated vectors in regions of low raw vector density, may not accurately predict the velocity flow at that point due to lack of data. In figure 13 , for example, vectors starting at axial locations less than $0.1 \mathrm{in}$. were extrapolated and are not accurate, and in figure 12 the interpolated results in the lower half of the channel were based on very few raw vectors.
The velocity on the upper and lower surfaces, travelling to the right, towards the inner wall, and the centerline velocity, travelling towards the outer wall, are the result of a pair of horizontally elongated vortices stacked on top of each other. The interpolated data also show some smaller scale vortices which are not obvious in the raw vector plots. These small vortices appear to be similar to the eddies observed in straight turbulent channel flow (see Schlichting [2]).

One subtle difference that can be seen between the $30^{\circ}$ and $90^{\circ}$ flow fields occurs where the centerline and wall velocities interact. At $30^{\circ}$, the centerline flow appears well developed and extends almost halfway to the upper and lower surfaces. At $90^{\circ}$ the mean velocity of the centerline flow is constrained towards the center of the channel. The small eddies that can be seen at the $30^{\circ}$ bend have grown in size and extend close to the centerline flow.

\section{Conclusions}

The secondary flow patterns in a curving rectangular bend of aspect ratio 5.0 were experimentally observed.

A new particle imaging velocimetry (PIV) technique was developed to analyze and quantify the observed cross-plane flow patterns. Combined with video tape recording of the seeded flowfield, this technique has been demonstrated as an effective method for obtaining flow velocity.

At a laminar flow rate, $\mathrm{Re}_{\mathrm{dh}}=1,000$, the classical vortex pair curvature effect flow structure was qualitatively observed. As the flow rate was increased, into turbulent transition, smaller scale vortices formed within the overall vortex pair secondary flow structure.

At a turbulent flow rate, $\mathrm{Re}_{\mathrm{dh}}=5,100$, qualitative observation was difficult, but the results from applying the PIV technique showed the development of a classical elongated secondary vortex pair structure at the earliest point of observation in the curve, $30^{\circ}$ from the start. Several smaller scale eddy vortices, similar to those observed in straight turbulent channel flow, were also apparent within the larger elongated vortices. These eddies strengthened as the flow progressed to $90^{\circ}$ into the curve. 
Although the Reynolds numbers in actual hydrogen coolant channels are two orders of magnitude greater than those in this experiment, observations of these low $\mathrm{Re}$, turbulent flows show the classical vortex pair flow structure and support the argument that the curvature effect heat transfer enhancement will persist in higher aspect ratio channels. Validation of the curvature heat transfer enhancement at accurate Reynolds numbers would require hot fire testing, as these conditions are beyond the capability of the current hardware.

\section{Acknowledgement}

The authors wish to thank Mr. Peter Quinn of NYMA, Inc. for his assistance in digitizing the video data and valuable discussions regarding this phase of the project.

\section{$\underline{\text { References }}$}

1. Carlile, J. A., Quentmeyer, R. J.,"An Experimental Investigation of High-AspectRatio Cooling Passages," NASA TN 105679, ALAA Paper 92-3154, 1992.

2. Schlichting, H., Boundary Layer Theory, McGraw Hill Book Company, 1979.

3. Humphrey, J. A .C., Launder, B. E., "Developing Turbulent Flow in Strongly Curved Passages of Square and Circular Cross Section," Final Technical Report for the Office of Naval Research, AD-A143 279, 1984.

4. Hendricks, R. C., Simon, F. F., "Heat Transfer to Hydrogen in a Curved Tube," presented at the ASME Meeting, Philadelphia, November, 1963.

5. Kacynski, K. J.,"Thermal Stratification Potential in Rocket Engine Coolant Channels," NASA TN 4378, 1992.

6. Yagley, J. A., Feng, J., Merkle, C. L.,"CFD Analyses of Coolant Channel Flowfields," AIAA Paper 93-1830, June 1993.

7. LeBail, F., Popp, M., "Numerical Analysis of High Aspect Ratio Cooling Passage Flow and Heat Transfer," AIAA Paper 93-1829, 1993.
8. Taylor, A. M. K. P., Whitelaw, J. H., Yiannekis, M., "Measurements of Laminar and Turbulent Flow in a Curved Duct with Thin Inlet Boundary Layers," NASA Contractor Report 3367, 1981.

9. Marko, K. A., Rimai, L., "Video Recording and Quantitative Analysis of Seed Particle Track Images In Unsteady Flows," Applied Optics, vol. 24, no. 21, November 1985.

10. Khalighi, B., Lee, Y. H., "Particle Tracking Velocimetry: An Automatic Image Processing Algorithm," Applied Optics, vol. 28, no. 20, October 1989.

11. Wernet, M. P., "Particle Displacement Tracking for PIV," NASA TM-104481, August, 1991.

12. Davis, W., Fox, R. W., "An Evaluation of the Hydrogen Bubble Technique for the Quantitative Determination of Fluid Velocities Within Clear Tubes," Journal of Basic Engineering, Trans. ASME, December 1967, pp. 771-781.

13. Tory, A. C., Haywood, K. H., "The Hydrogen Bubble Technique of Flow Visualization: Factors Affecting Bubble Size and Buoyancy," Fluids Engineering Conference, ASME Paper 71-FE-36, May 1971, Pittsburgh, Pa.

14. Diemunsch, G. and Prenel, J.P., "A Compact Light Sheet Generator," Optics and Laser Technology, vol. 14, no. 3, June 1987.

15. Foley, T. A., "Scattered Data Interpolation and Approximation With Error Bounds", Computer Aided Geometric Design, 3, 163177, 1986. 


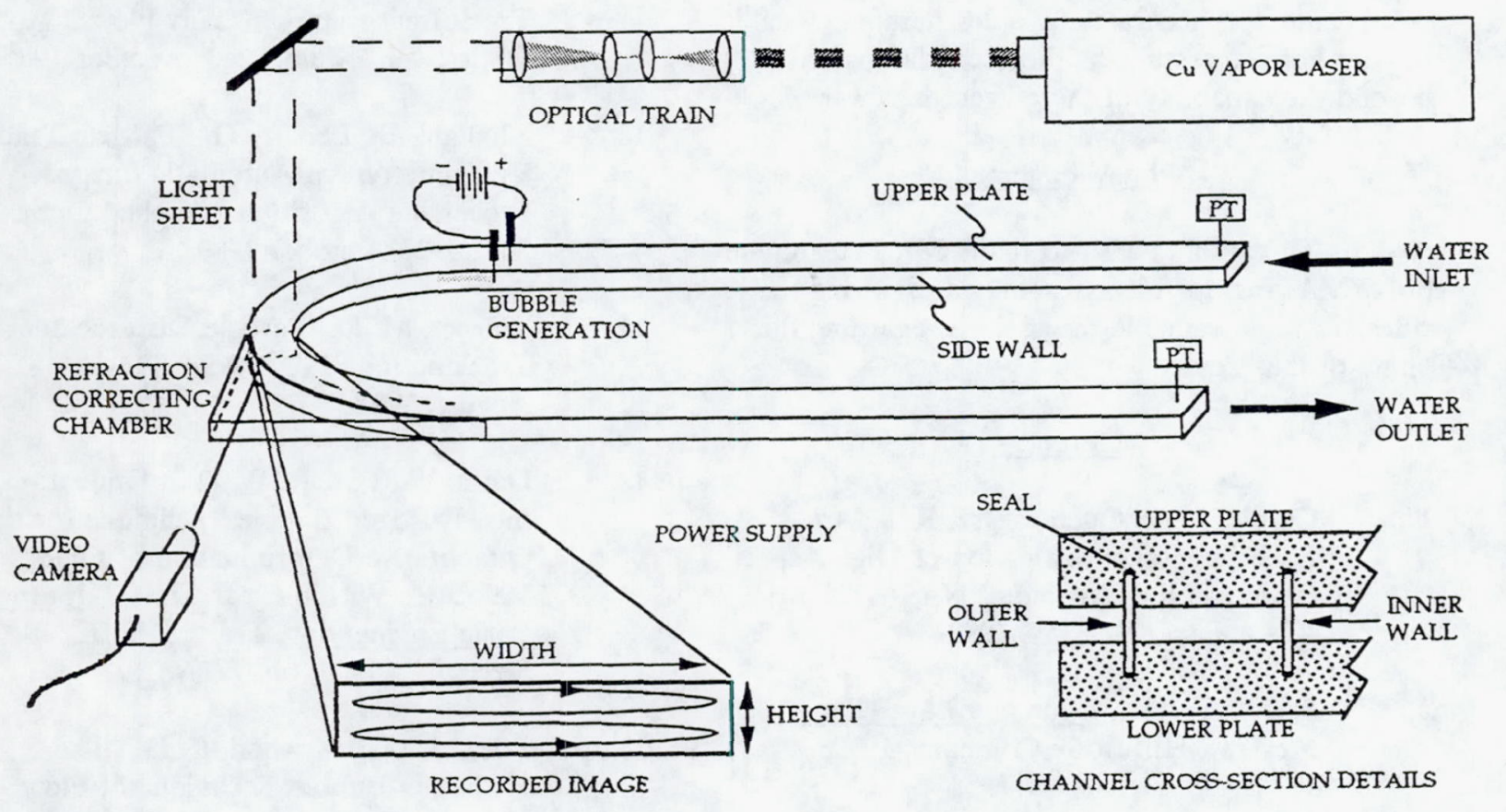

Figure 1. Components of the Plexiglass High Aspect Ratio Visualization Rig. 


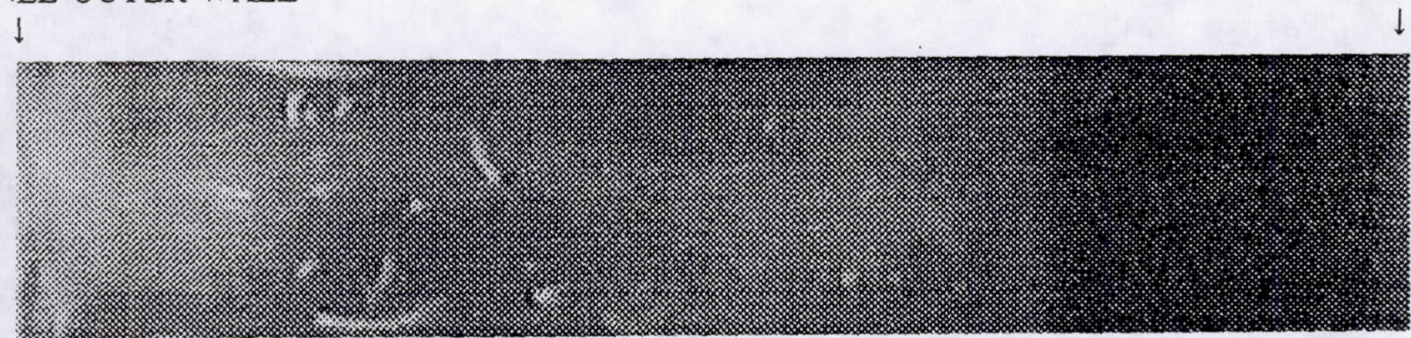

Figure 2. Digitized Image of Raw Video Data.

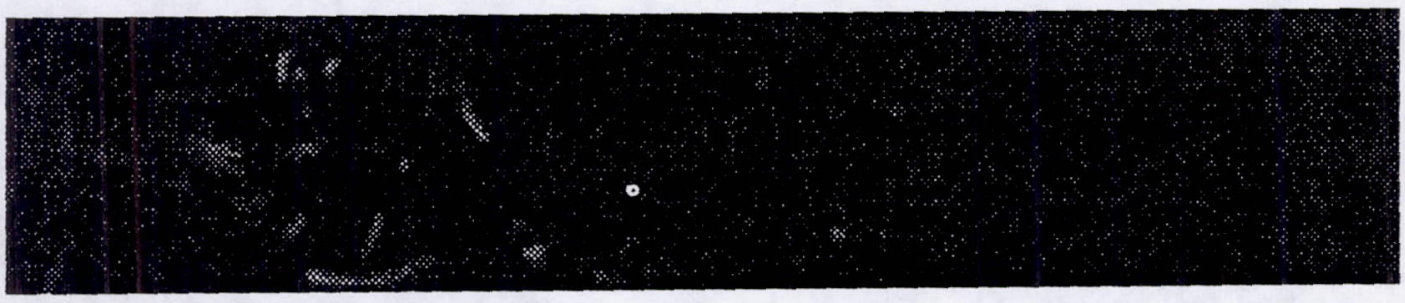

Figure 3. Enhanced Digitized Image.

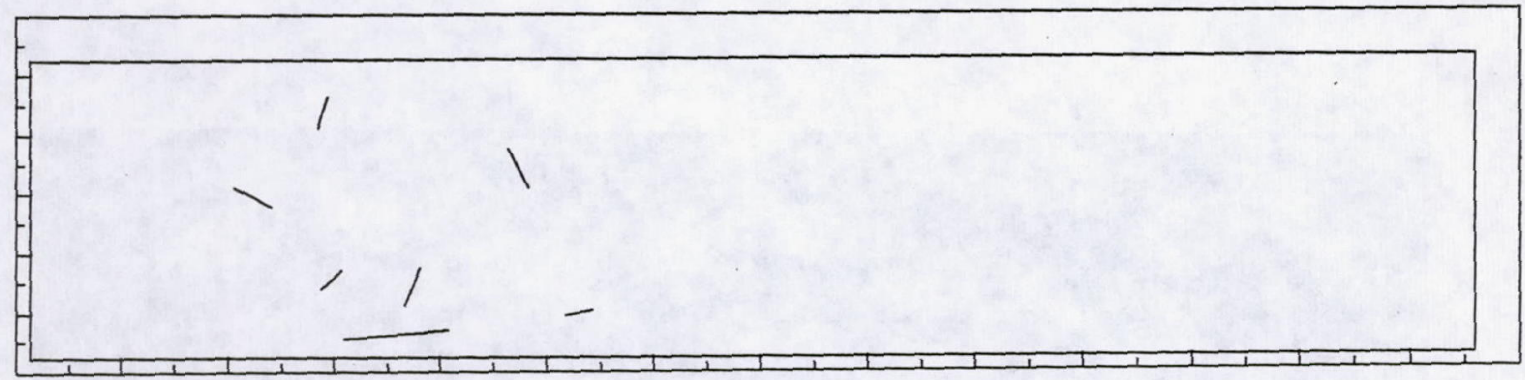

Figure 4. Bubble Streaks Resolved from Digitized Video Image. 


\section{CHANNEL OUTER WALL}

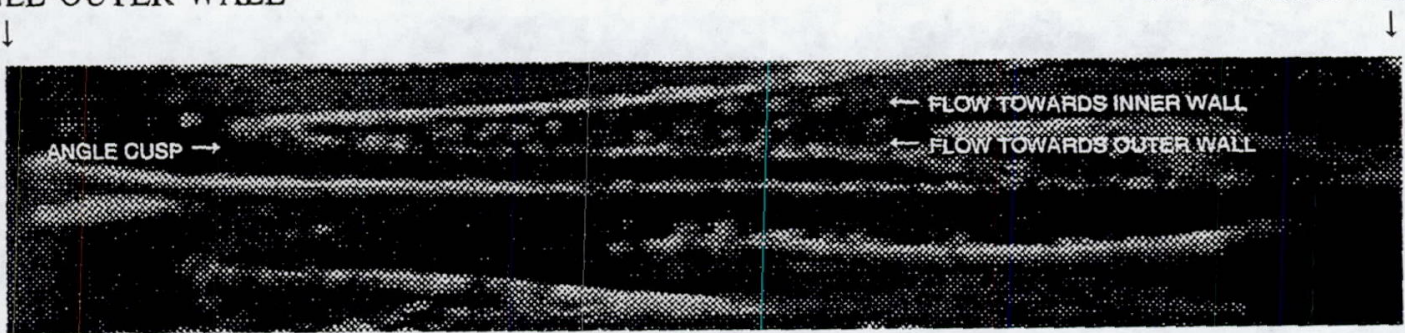

Figure 5. Digitized Video Frame: $R e=1,000$ at $90^{\circ}$ into the Bend.

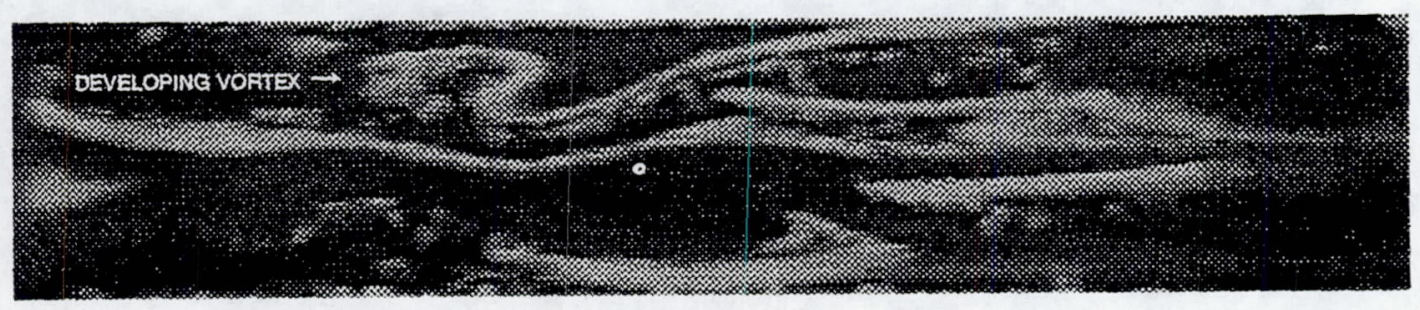

Figure 6. Digitized Video Frame: $\operatorname{Re}=1,700$ at $90^{\circ}$ into the Bend.

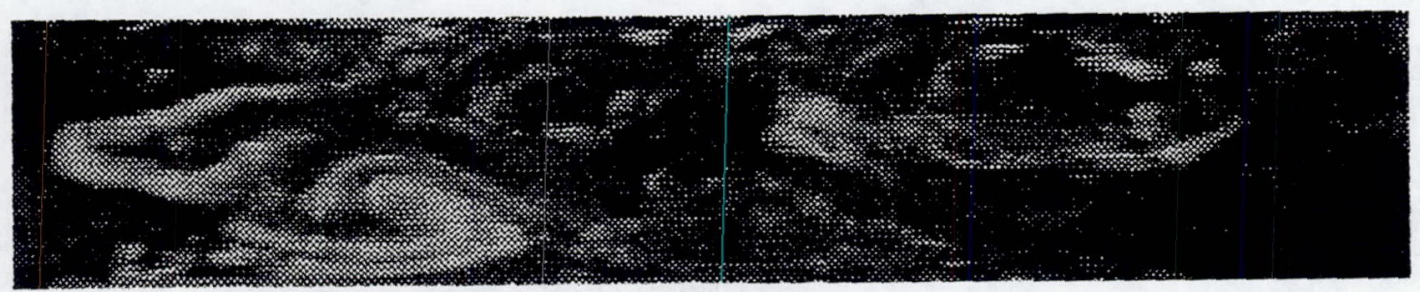

Figure 7. Digitized Video Frame: $R e=3.400$ at $90^{\prime \prime}$ into the Bend. 


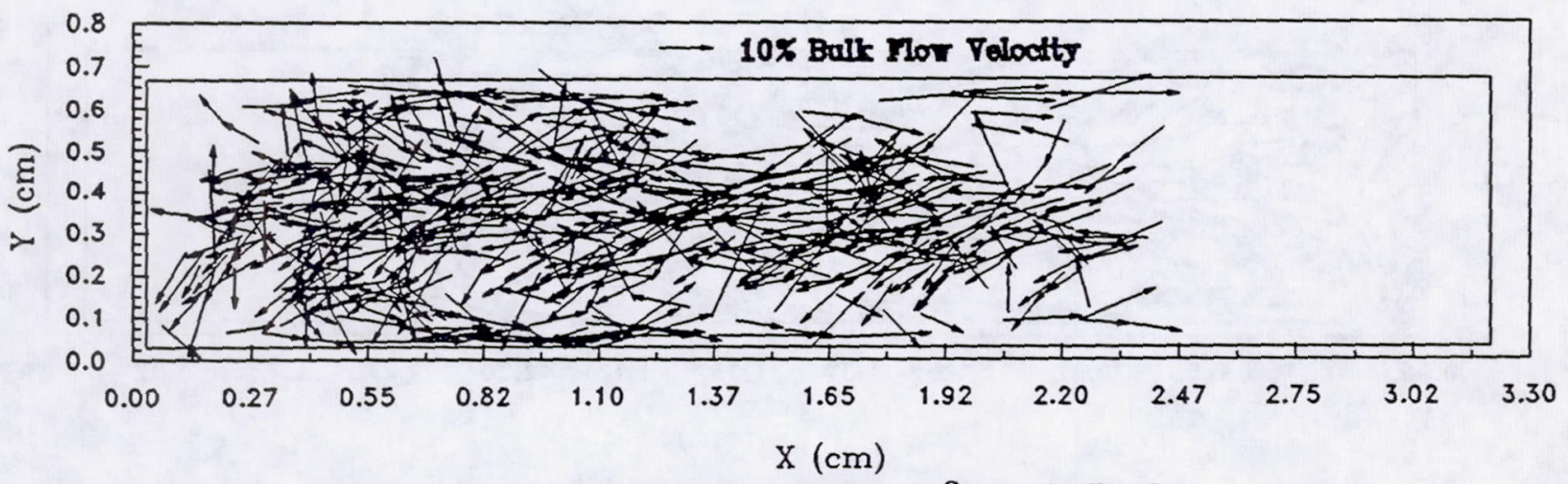

Figure 8. Raw Data: $\operatorname{Re}=5,100$ at $30^{\circ}$ into the Bend.

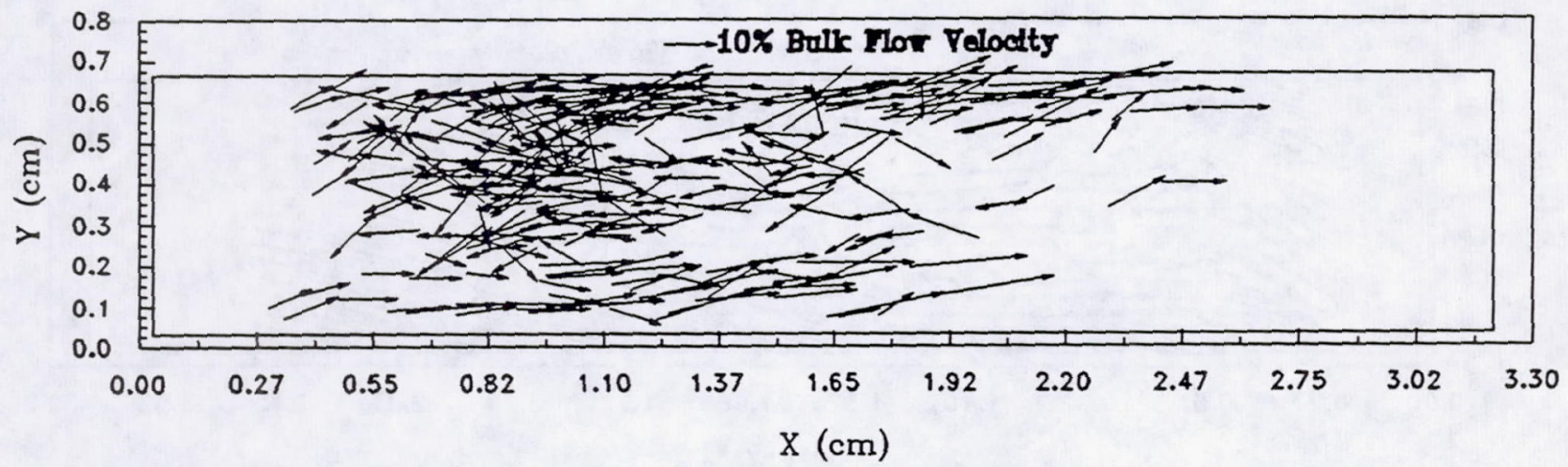

Figure 9. Raw Data : $\operatorname{Re}=5,100$ at $60^{\circ}$ into the Bend.

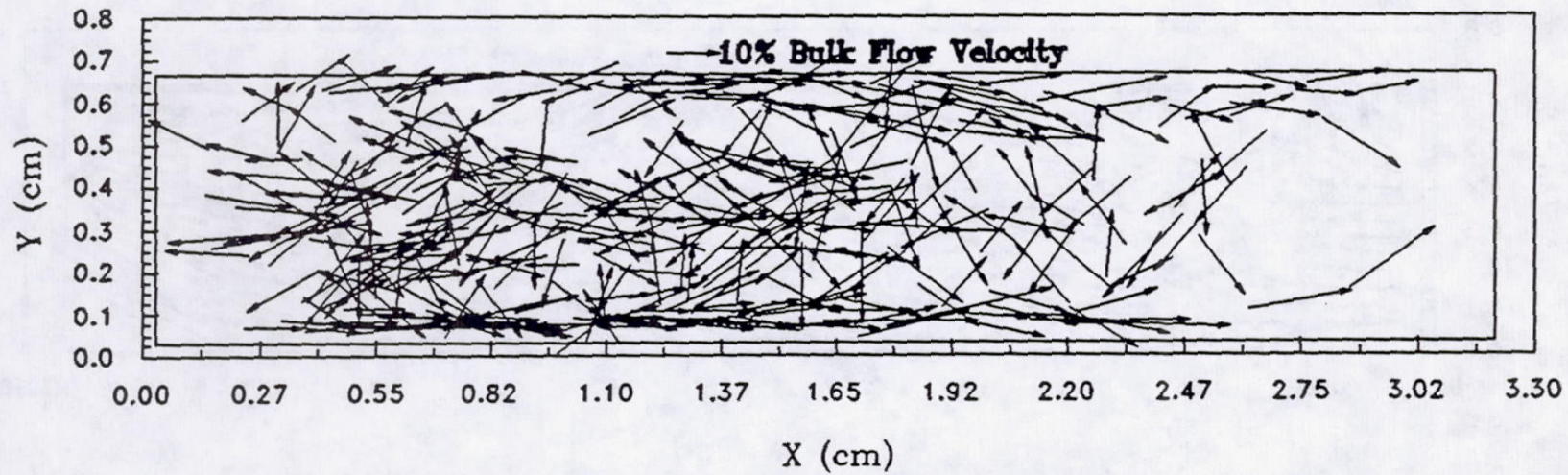

Figure 10. Raw Data: $\operatorname{Re}=5,100$ at $90^{\circ}$ into the Bend. 


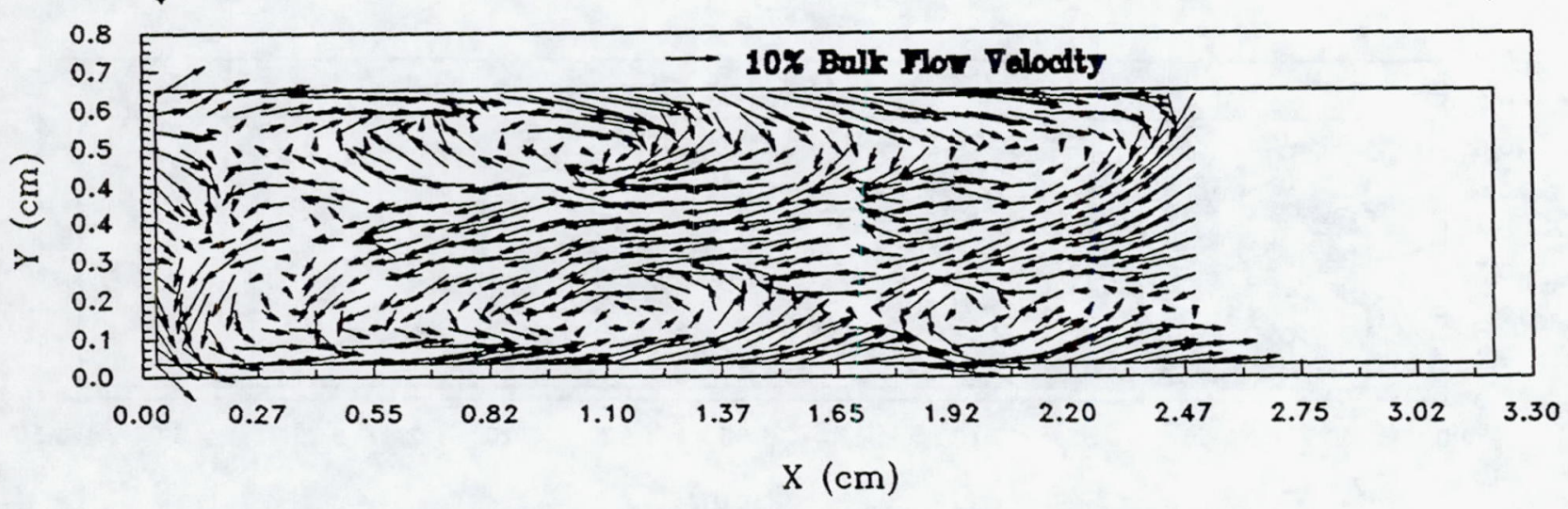

Figure 11. Interpolated Data : $\operatorname{Re}=5,100$ at $30^{\circ}$ into the Bend.

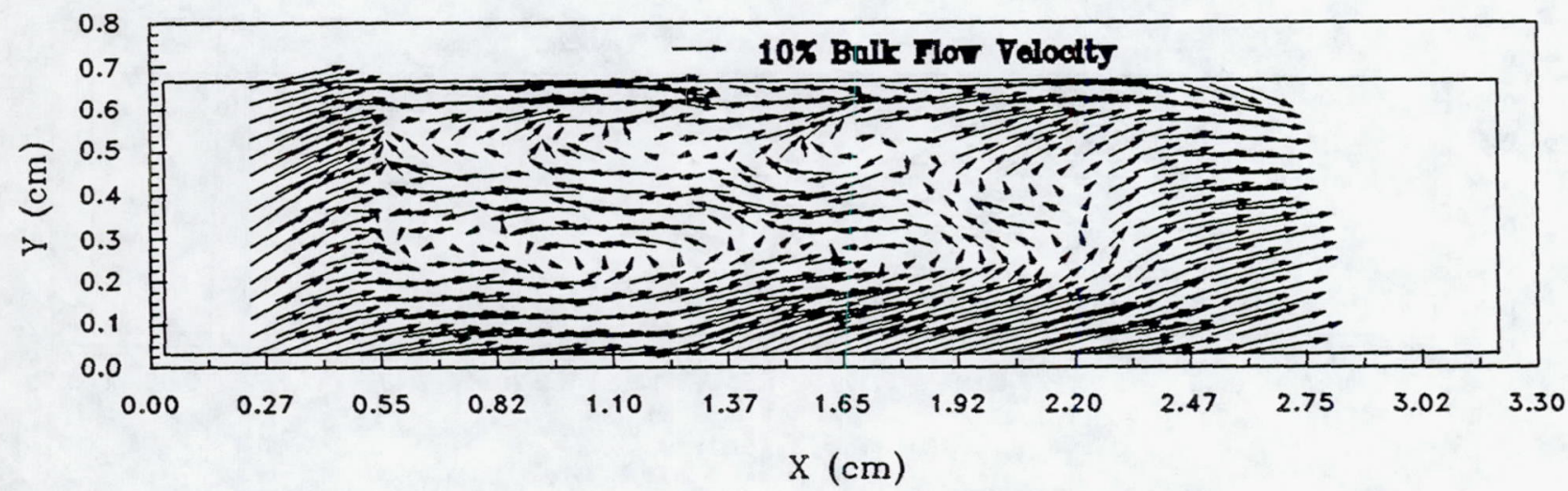

Figure 12. Interpolated Data : $\operatorname{Re}=5,100$ at $60^{\circ}$ into the Bend.

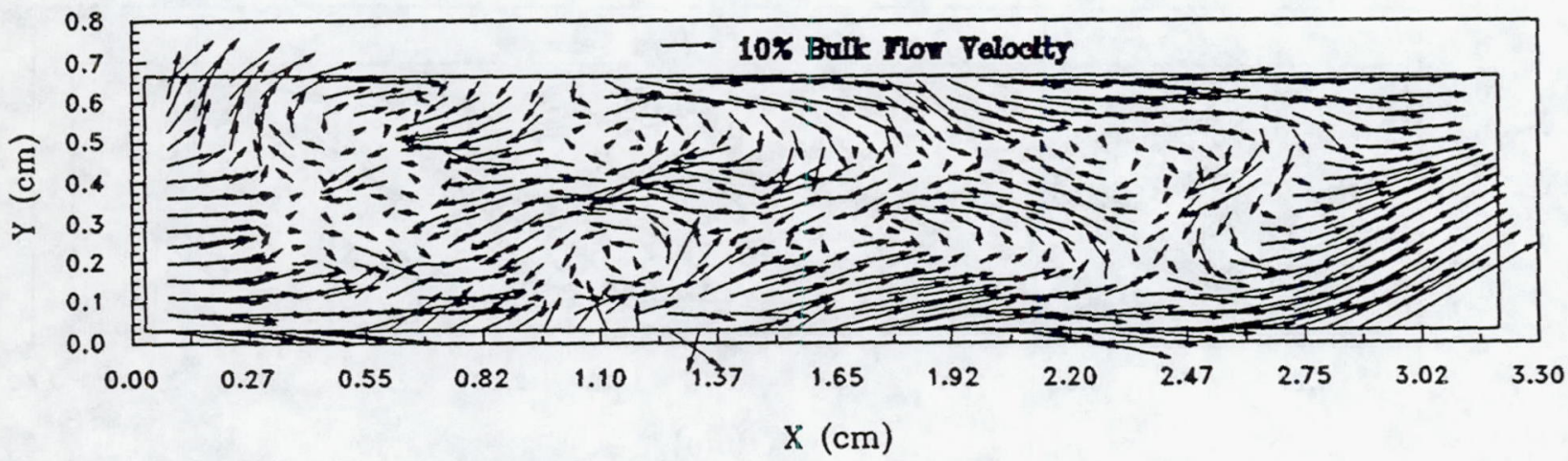

Figure 13. Interpolated Data : $\operatorname{Re}=5,100$ at $90^{\circ}$ into the Bend. 


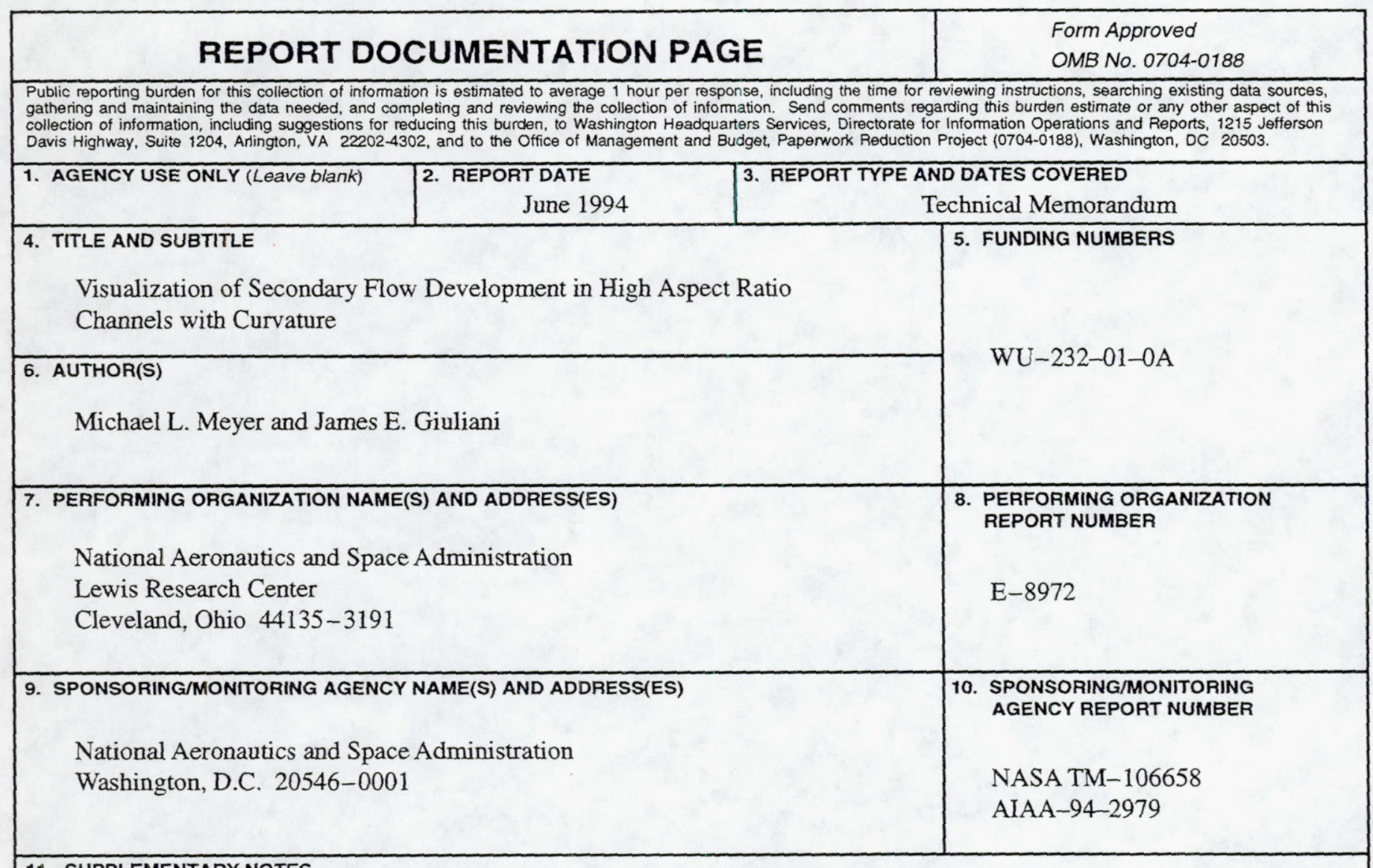

11. SUPPLEMENTARY NOTES

Prepared for the 30th Joint Propulsion Conference cosponsored by the AIAA, ASME, SAE, and ASEE, Indianapolis, Indiana, June 27-29, 1994. Michael L. Meyer, NASA Lewis Research Center, and James E. Giuliani, Ohio Aerospace Institute, 22800 Cedar Point Road, Brook Park, Ohio 44142. Responsible person, Michael L. Meyer, organization code 5310, (216) 433-7492.

12a. DISTRIBUTIONAVAILABILITY STATEMENT

Unclassified - Unlimited

Subject Categories 15, 20, and 34

13. ABSTRACT (Maximum 200 words)

The results of an experimental project to visually examine the secondary flow structure that develops in curved, high aspect-ratio rectangular channels are presented. The results provide insight into the fluid dynamics within high aspect ratio channels. A water flow test rig constructed out of plexiglass, with an adjustable aspect ratio, was used for these experiments. Results were obtained for a channel geometry with a hydraulic diameter of $10.6 \mathrm{~mm}(0.417 \mathrm{in}$.), an aspect ratio of 5.0, and a hydraulic radius to curvature radius ratio of 0.0417 . Flow conditions were varied to achieve Reynolds numbers up to 5,100. A new particle imaging velocimetry technique was developed which could resolve velocity information from particles entering and leaving the field of view. Time averaged secondary flow velocity vectors, obtained using this velocimetry technique, are presented for $30^{\circ}, 60^{\circ}$, and $90^{\circ}$ into a $180^{\circ}$ bend and at a Reynolds number of 5,100 . The secondary flow results suggest the coexistence of both the classical curvature induced vortex pair flow structure and the eddies seen in straight turbulent channel flow.

\section{SUBJECT TERMS}

Flow visualization; Secondary flow; Turbulent flow; Water flow; Curvature; Channel flow; Image analysis; Velocity fields; Velocity measurement; High aspect ratio

\begin{tabular}{|c|c|c|}
\hline $\begin{array}{c}\text { 17. SECURITY CLASSIFICATION } \\
\text { OF REPORT } \\
\text { Unclassified }\end{array}$ & $\begin{array}{c}\text { 18. SECURITY CLASSIFICATION } \\
\text { OF THIS PAGE } \\
\text { Unclassified }\end{array}$ & $\begin{array}{c}\text { 19. SECURITY CLASSIFICATION } \\
\text { OF ABSTRACT } \\
\text { Unclassified }\end{array}$
\end{tabular}

